\title{
Overweight and Obese Have Similar Burden of Hip Fracture as Normal Weight Older Adults
}

\author{
Jennifer T. Lloyd, PhD, MA, MS ${ }^{1,2}$, Shari R. Waldstein, $P h D^{3,4}$, \\ Marc C. Hochberg, MD, MPH ${ }^{5}$, Denise L. Orwig, PhD ${ }^{4,5}$, and Dawn E. Alley, $P h D^{7}$
}

${ }^{1}$ Center for Medicare \& Medicaid Innovation, Baltimore, MD, USA; ${ }^{2}$ Centers for Medicare \& Medicaid Services, Baltimore, MD, USA; ${ }^{3}$ Department of Psychology, University of Maryland, Baltimore County, Baltimore, MD, USA; ${ }^{4}$ Doctoral Program in Gerontology, University of Maryland, Baltimore and Baltimore County, Baltimore, MD, USA; ${ }^{5}$ Department of Epidemiology and Public Health, University of Maryland, Baltimore, MD, USA.

$\mathrm{J}$ Gen Intern Med 34(11):2333-5

DOI: $10.1007 / \mathrm{s} 11606-019-05151-y$

(c) Society of General Internal Medicine (This is a U.S. government work and not under copyright protection in the U.S.; foreign copyright protection may apply) 2019

\section{INTRODUCTION}

The association between body weight and fracture risk is a significant public health concern as the majority of older adults are overweight or obese and have low mineral density. ${ }^{1}$ Osteoporotic fractures are projected to cost $\$ 25$ billion in health care spending annually by 2025 , with hip fractures accounting for over $70 \%$ of these costs. ${ }^{2}$ Post-fracture spending is $25 \%$ higher among obese individuals. ${ }^{3}$

Numerous studies have found that overweight and obesity protect against risk of hip fracture, relative to normal or underweight. ${ }^{1}$ However, Nielson et al. (2012) showed that close to half (46\%) of hip fractures occurred in overweight and obese older adults using data from the 1970 to $1980 \mathrm{~s} .{ }^{1}$ Even though heavier individuals may have a lower relative risk of hip fracture, they may have a similar absolute risk, particularly as the prevalence of obesity continues to increase among the aging population. The aims of this paper are to provide updated estimates of the incidence of hip fracture and to demonstrate that the current burden of hip fracture occurs among heavier older adults.

\section{METHODS}

We linked survey data from two nationally representative studies to claims data from the Center for Medicare \& Medicaid Services. Both samples were restricted to individuals who were alive and 65 years of age and older during the baseline year and were enrolled in Medicare Parts A and B (not in Medicare Advantage)

$\overline{\text { Prior Presentation This work was not previously presented at a }}$ conference.

Published online July 19, 2019 throughout the follow-up period. Underweight beneficiaries were not presented as they represented a small proportion of the overall study samples $(2.2 \%$ in NHANES III 1988-1994 and 0.8\% in MCBS 2006). Baseline survey data from 2790 (weighted $N=$ $19,788,041$ ) US adults (aged $\geq 65$ ) examined in the National Health and Nutrition Examination Survey (NHANES) III, 1988-1994, were linked to Medicare claims and enrollment data (1991-2007). Baseline survey data from 4332 (weighted $N=12,064,136$ ) US community-dwelling adults (aged $>65$ ) interviewed in the 2006 Medicare Current Beneficiary Survey (MCBS) were linked to Medicare claims and enrollment data (2007-2017).

Body mass index (BMI) was categorized as underweight $(<18.5)$, normal weight (18.5-24.9), overweight (25.0-29.9), or obese $(\geq 30.0)$. BMI was measured (NHANES) or self-reported (MCBS) during the baseline survey year. Self-reported height and weight were corrected for following methods described by Cawley and Burkhauser (2006). ${ }^{4}$ Incident hip fracture cases were identified using ICD-9 (e.g., code 820) and ICD-10 codes in Medicare claims.

The proportion of individuals within each BMI group from the NHANES III and MCBS (2006) samples is displayed in Figure 1. The unadjusted incidence of hip fractures per 10,000 persons by BMI group for both surveys is reported in Figure 2. Survey weights were used to generate nationally representative estimates.

\section{RESULTS}

Obese older adults represent a third of the aging population in the MCBS (2006) sample but only $23 \%$ in NHANES III (1988-1994) sample; normal weight older adults now represent only $26 \%$ of the population (Fig. 1).

Normal weight older adults had the highest incidence of hip fracture in NHANES III (1988-1994); more recent data from MCBS suggest similar rates across BMI groups (Fig. 2). Currently, the majority of hip fractures $(65 \%)$ occur among overweight and obese older adults (Fig. 2). 

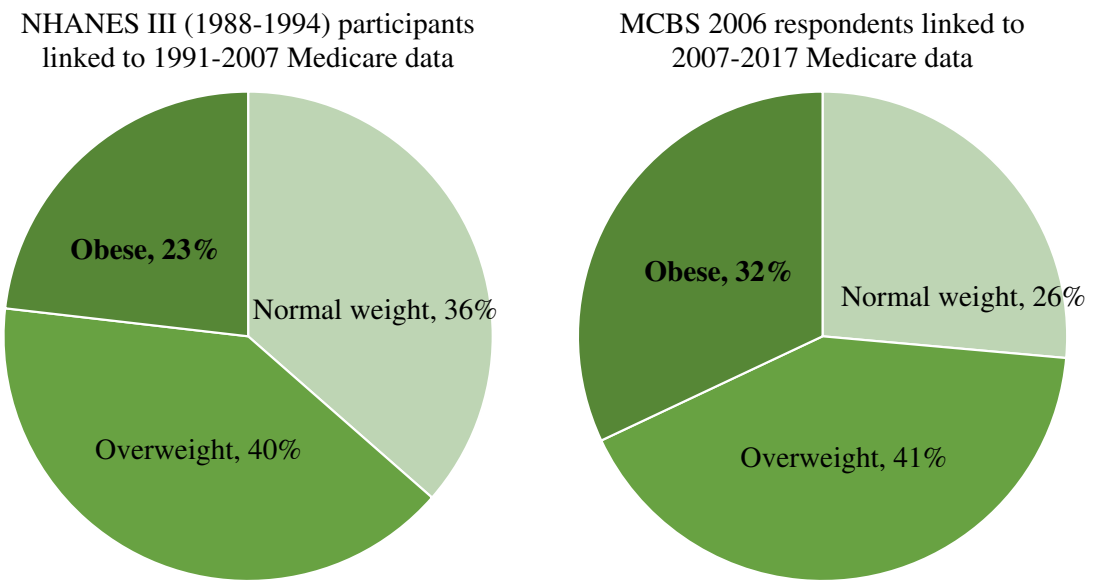

Figure 1 Twenty-three percent of older adults were obese in the National Health and Nutrition Examination Survey (NHANES) III (19881994); shifting weight distributions in newer cohorts of older adults show that $32 \%$ of older adults were obese in more recent years using data from Medicare Current Beneficiary Survey (MCBS; 2006). Notes: NHANES III (1988-1994) participants were linked to Medicare claims and enrollment data (weighted $N=19,788,041$ ) from 1991 to 2007. MCBS respondents in 2006 were linked to Medicare claims and enrollment data (weighted $N=12,064,136$ ) from 2007 to 2017 .

\section{DISCUSSION}

These findings suggest a large segment of older adults may be overlooked for hip fracture prevention and screening. ${ }^{1}$ The relationship between body composition and risk of fracture is complicated; this analysis is not intended to imply a causal relationship nor is it intended to speak to the potential mechanisms underlying these trends. ${ }^{1}$ Recent cohorts of older adults have spent larger proportions of their lives obese, ${ }^{5}$ resulting in early development of disability and chronic disease, such as cardiovascular disease, diabetes, and osteoarthritis, further increasing risk for falls, bone loss, and fracture. ${ }^{1}$
BMI is an imprecise measure of body composition; better measures of fat and muscle mass reflect the negative aspects of excess weight. For example, subgroups of heavier individuals with morbid obesity, sarcopenia, and/ or abdominal obesity are at increased risk for falls and fracture and thus are important subgroups to target with interventions. ${ }^{1}$ These subgroups share underlying mobility limitations, which may provide a more pragmatic marker of fracture risk than weight as well as an opportunity to reduce the risk. ${ }^{6}$

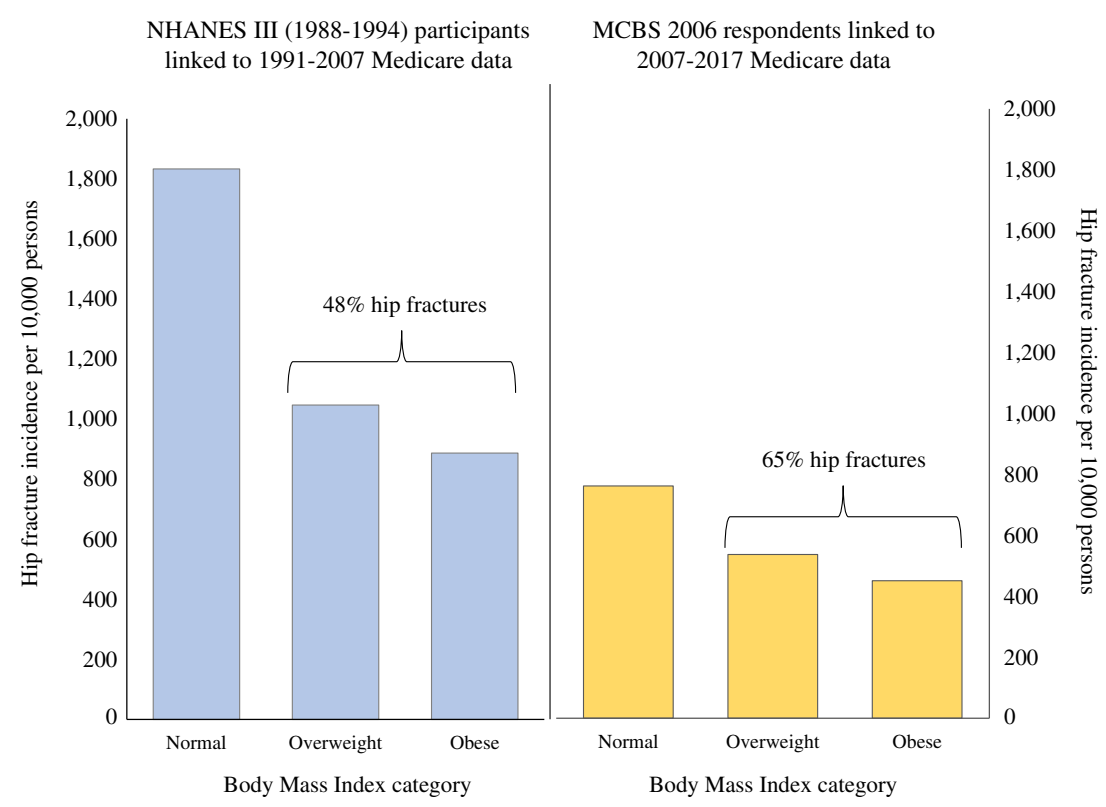

Figure 2 Hip fracture incidence was highest among normal weight older adults in National Health and Nutrition Examination Survey (NHANES) III (1988-1994); more recent data from Medicare Current Beneficiary Survey (MCBS; 2006) suggest similar rates across BMI groups. Currently, overweight and obese older adults account for the majority of hip fractures (65\%). Notes: NHANES III (1988-1994) participants were linked to Medicare claims and enrollment data (weighted $N=\mathbf{1 9 , 7 8 8 , 0 4 1}$ ) from 1991 to 2007. MCBS respondents in 2006 were linked to Medicare claims and enrollment data (weighted $N=12,064,136)$ from 2007 to 2017. 
Acknowledgments: We would like to thank the National Center for Health Statistics and Research Data Center for their assistance with accessing restricted data files. We would also like to thank Suzanne G. Wensky for instructive comments on an earlier draft of this manuscript.

Corresponding Author: Jennifer T. Lloyd, PhD, MA, MS; Centers for Medicare \& Medicaid Services, 7500 Security Blvd., Mail Stop WB-06 05, Baltimore, MD 21244, USA (e-mail: Jennifer.lloyd@cms.hhs.gov).

Funders This work was supported by grants from the National Institutes of Health (T32 4G000262 and R01AG028556, Jennifer Lloyd), the Robert Wood Johnson Foundation Health and Society Scholars program (Dawn Alley), as well as from the Claude D. Pepper Older Americans Independence Center (P3OAG028747, Shari Waldstein).

\section{Compliance with Ethical Standards:}

Conflict of Interest: The authors declare that they do not have a conflict of interest.
Disclaimer: The statements contained herein are those of the authors and do not necessarily reflect the views or policies of the Centers for Medicare \& Medicaid Services.

\section{REFERENCES}

1. Nielson CM, Srikanth P, Orwoll E. Obesity and fracture in men and women: An epidemiologic perspective. J Bone Miner Res. 2012;27(1):1-10.

2. Burge R, Dawson-Hughes B, Solomon DH, et al. Incidence and economic burden of osteoporosis-related fractures in the United States, 2005-2025. J Bone Miner Res. 2007;22(3):465-475.

3. Schousboe JT, Paudel ML, Taylor BC, et al. Pre-fracture individual characteristics associated with high total health care costs after fracture. Osteoporos Int. 2017; 28(8): 889-899.

4. Cawley J, Burkhauser RV. Beyond BMI: the Value of More Accurate Measures of Fatness and Obesity in Social Science Research. Cambridge: National Bureau of Economic Research; 2006:1-50.

5. Leveille SG, Wee CC, Iezzoni LI. Trends in obesity and arthritis among baby boomers and their predecessors, 1971-2002. Am J Public Health. 2005;95(9):1607-1613.

6. Himes CL, Reynolds SL. Effect of Obesity on Falls, Injury, and Disability. JAGS. 2012; 60:124-29.

Publisher's Note: Springer Nature remains neutral with regard to jurisdictional claims in published maps and institutional affiliations. 